\title{
WIRELESS-BASED ENERGY AUTONOMOUS TIRE PRESSURE MONITORING SYSTEM
}

\author{
Jens Warmuth ${ }^{1,2}$, Thomas Zimmermann ${ }^{3}$, Matthias Schreiter ${ }^{2}$, Alexander Frey ${ }^{4}$, Ingo Kuehne ${ }^{5}$ \\ Jens.Warmuth@gmx.de
}

${ }^{1}$ Munich University of Applied Science, Department of Applied Sciences and Mechatronics, Munich, Germany

${ }^{2}$ Siemens AG, Corporate Technology, Research \& Technology Center, Munich, Germany

${ }^{3}$ Ludwig-Maximilians-University Munich, Institute for Informatics, Munich, Germany

${ }^{4}$ University of Applied Sciences Augsburg, Faculty of Electrical Engineering, Augsburg, Germany

${ }^{5}$ Heilbronn University, Faculty of Engineering and Business Administration, Heilbronn, Germany

\begin{abstract}
:
This paper presents a new approach to energy harvesting for use in sensor applications. It explores the possibility of using low frequency high power forces to generate energy. The subject is approached by showing the feasibility of an autonomous tire pressure monitoring system (TPMS). Any section of a car's tire gets a periodic bump when with every rotation the tire is dented by the ground. The developing forces are converted into electricity by use of a piezoelectric generator. Because of the lower excitation rate a special power-management circuit is required to convert the generated energy into a form usable by the sensor system. The sensor itself is specially designed to run extremely energy efficient and to utilize the stored energy of several generator excitations while remaining in an inert state in between. When power is provided it takes a pressure measurement and links up to a base station to deliver the collected data. By use of this approach it becomes possible to provide the car's driver with real time safety relevant information without the need for any maintenance after the system has been installed.
\end{abstract}

Key words: MEMS, Energy-Harvesting, Autonomous Sensor Node, Piezoelectricity, Tire Pressure Monitoring System

\section{Introduction}

Wireless sensor nodes are used in a broad field of application. At present the nodes are powered by energy storage units like batteries or accumulators. They are however limited in their storage capability so they have to be replaced or recharged regularly. As a consequence they are not feasible for long term usage. The goal is an energy autonomous sensor to lift time restrictions and to minimize corresponding service effort. To achieve this the batteries have to be changed with power converters which harvest their energy from the surrounding environment. Because of the restrictions in sustainability and the capacity limitations of fossil and nuclear sources renewable energy has to be used. Such sources are:

- Electromagnetic radiation sources: light and radio waves

- Thermal sources

- Mechanical sources: water, wind and vibration. 


\section{The Pressure Monitoring System}

The automotive sector is one of the major fields of application for this technology. A mediumsized vehicle is typically equipped with about 60 sensors which are used for comfort and security applications. This number is about to increase significantly in the near future as more innovative functions are added.

Conventional tire pressure monitoring systems (TPMS) are either placed at the wheel rim or the inner lining of the tire as shown in Fig.1 [1]. This conventional nodes are today powered by batteries. A future goal is place the TPMS on the inner line in order to get an "intelligent" tire. This also means exchanging the storage devices with an energy conversion module because of system mass restrictions.

It has been established that for an efficient use (a car driving $15 \mathrm{~km} / \mathrm{h}$ or faster and a sensor giving a reading every 150 meters) the system will be content with $6 \mu \mathrm{W}$ of electric power [2].

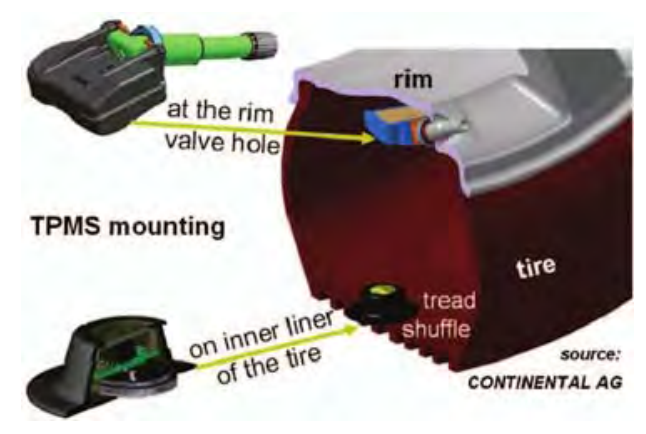

Fig. 1. TPMS mounting options [1]

\section{Piezoelectric Energy Harvester}

The here described energy harvester is placed at the inner lining of the tire. Every time it enters the tread shuffle the generator gets a powerful hit. The force applied on it is to be used to convert this mechanical energy into electricity. Several design options have been considered and evaluated. The best concept was shown to be a piezoelectric cantilever beam.

When the free end of a cantilever is deflected downwards it results in tensile stress in its upper and compression stress in its lower part. Mechanical stresses can be converted to electricity by a piezoelectric material. However if the whole of the beam consists of one single piezoelectric crystal the opposing stresses would cancel each other out. To counteract this problem there are two possibilities either a separation material must be placed in the neutral fiber or half the piezoelectric layer has to be outside the neutral fiber using an additional carrier layer. For the generators described here the carrier approach was chosen because of the relative constructing simplicity.

If a strong force deflects the free end of the beam it then continues to decaying oscillate until again deflected or come to a stop. With every oscillating period the piezoelectric layer transforms part of the mechanical energy stored in the whole cantilever into electricity. At the next deviation of the beam the piezoelectric is again loaded with mechanical energy from the silicon-based cantilever. It then can again transform this mechanical energy into electric energy. So, the carrier layer operates as a spring where mechanical energy is efficiently stored.

To archive maximum power conversion it's imperative that stress is distributed evenly throughout the length of the cantilever. Therefore the beam is designed as a triangle [3]. The Generator is shown in Fig. 2.

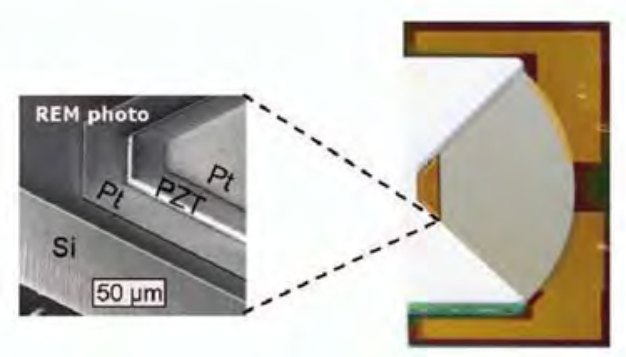

Fig. 2. MEMS-based piezoelectric energy harvester

After the construction of the generator it has been evaluated. To generate the maximum power from the generator it has to work in state of load adaptation meaning it's internal impedance must be equal to the connected load [4]. Since the internal impedance is fixed the load has to be optimized. The electrical output power was measured over a number of resistive loads. The result is shown in Fig. 3. 


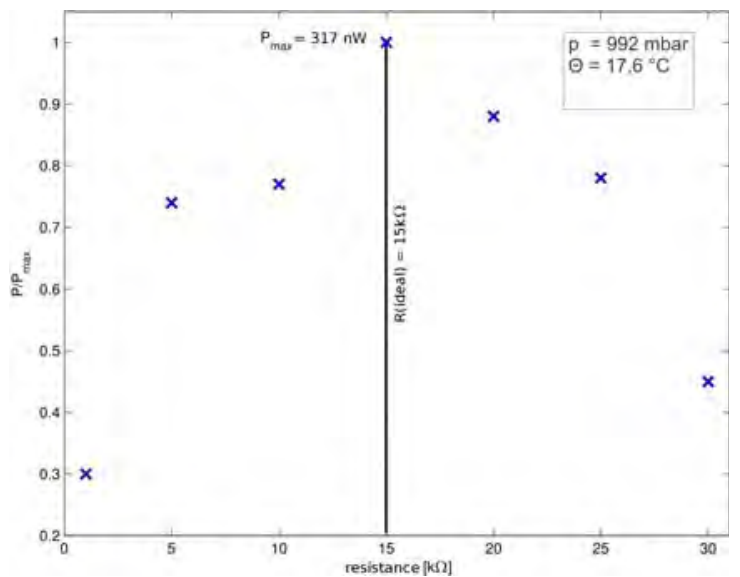

Fig. 3. Impedance matching by an external resistive load

The following results are all measured using the optimum load of $15 \mathrm{k} \Omega$. Next is was important to measure the influence of

the ambient pressure on the generator. It could be possible that higher pressure levels in a tire have a strong dampening effect on the generator. This would have a negative effect on its power conversion capability and could distort the results taken at atmospheric pressure. Since no overload pressure measuring station was available a partial vacuum was used to get a general overview of the generators pressure sensitivity. To evaluate the effect of the different pressure levels the quality factor was used. The results are shown in Fig. 4.

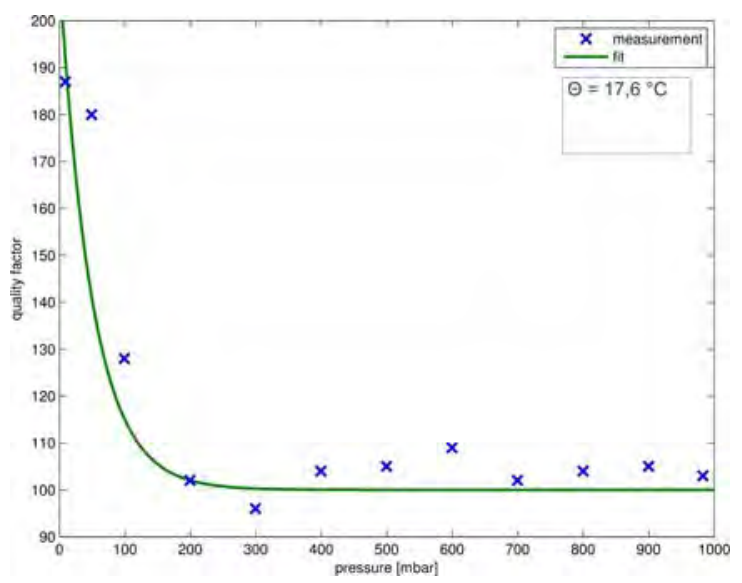

Fig. 4. Pressure sensitivity of the quality factor
It's clearly visible that there is no significant change in the quality factor starting at at pressure of 200 mbar. One can therefore conclude that it will be similar at higher pressures inside a cars tire.

Given this two results it is now possible to evaluate the generator efficiency and conclude similar behavior in the working environment. Beside the efficiency the coupling factor was also evaluated to be comparable to results of previous calculations. The coupling factor and the efficiency of the generator for a wide band of deflection rates are shown in Fig. 5.

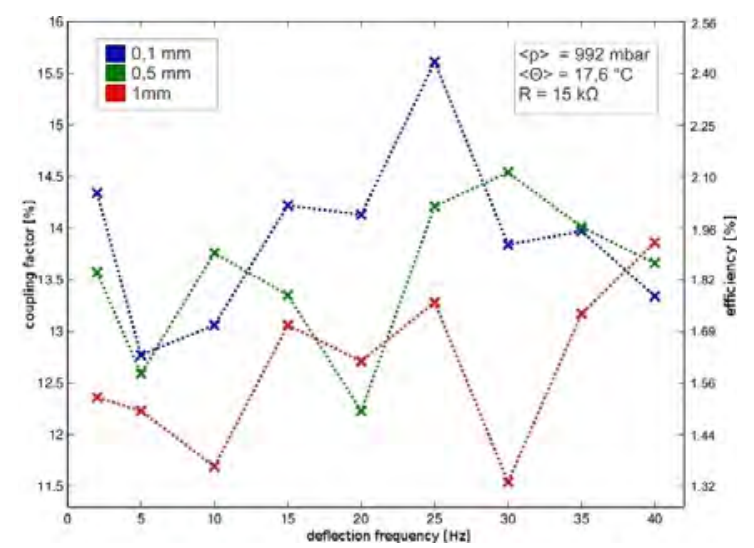

Fig. 5. Coupling factor and efficiency of the generator

\section{ASIC}

The generators electrical output signal is still in a form hard to process by a sensor and even less applicable for storage. For this purpose a special application-specific integrated circuit (ASIC) was designed [5]. Itself and its circuit diagram are shown in Fig. 6.

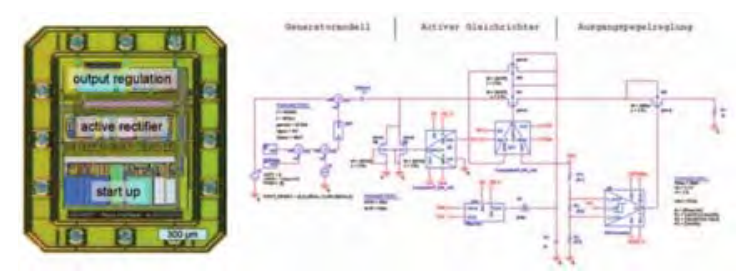

Fig. 6. ASIC and its circuit diagram 
The ASIC rectifies the decaying oscillating to a direct current which is stored on a capacity. First the rectification is achieved passively by diodes. All other components of the ASIC and the load are entirely disabled. The passive rectification will be taken over by an active rectifier when the energy level in the buffer will exceed a predetermined level (approx. 1.6 V). The energy extraction by the active rectification is evidently more efficient because the energy loss across the active switches is much lower than the loss on passive diodes. The load still remains disconnected at this time. When the capacitor's voltage reaches approximately $3.5 \mathrm{~V}$ the ASIC switches from loading the capacity to releasing the stored energy to the sensor. At that point the voltage at the capacity starts to decline as the sensor is gathering and transmitting data. At app. $2.8 \mathrm{~V}$ the ASIC switches back to loading the capacity and the sensor goes back to sleep until it is reactivated at the beginning of the next working phase. The whole process is shown in Fig. 7 .

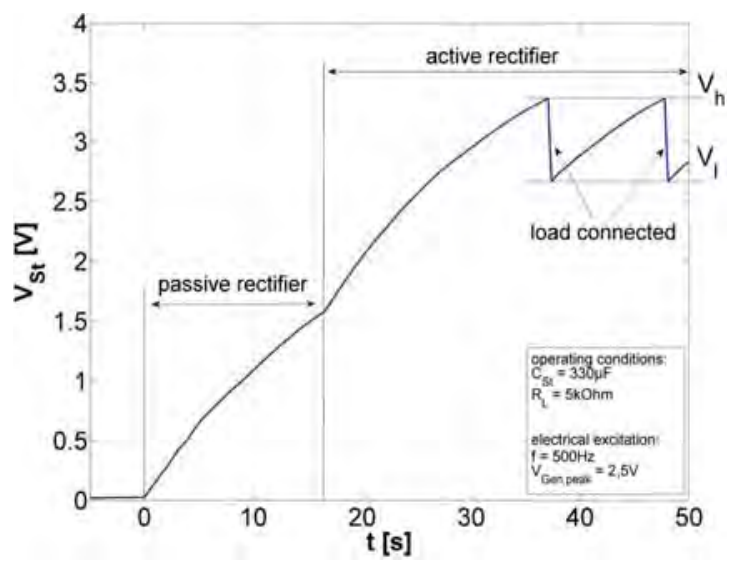

Fig. 7. ASIC capacity loading curve

\section{Energy Harvesting Module}

At this point in its development the discussed generator still lacks the capability to produce the voltage necessary to use the ASIC. Therefore a functional similar bulk generator was used to evaluate a complete system. With it it was not only possible to evaluate the ASICs efficiency under realistic conditions but also to get a preview of the efficiency of the whole energy harvesting module. The efficiency of the ASIC was measured to about $50 \%$.

\section{Autonomous Sensor Node}

The here exemplarily presented autonomous sensor node consists of the previously descripted energyy harvesting module, a microcontroller, a temperature sensor including an interface and a radiofrequency front end [6]. The single parts were integrated onto a printed circuit board (PCB). Fig. 8 shows the complete sensor node on the PCB. This type of sensor node consumes significantly more energy compared to a simple TPMS which is a useful benchmark for evaluation purposes.

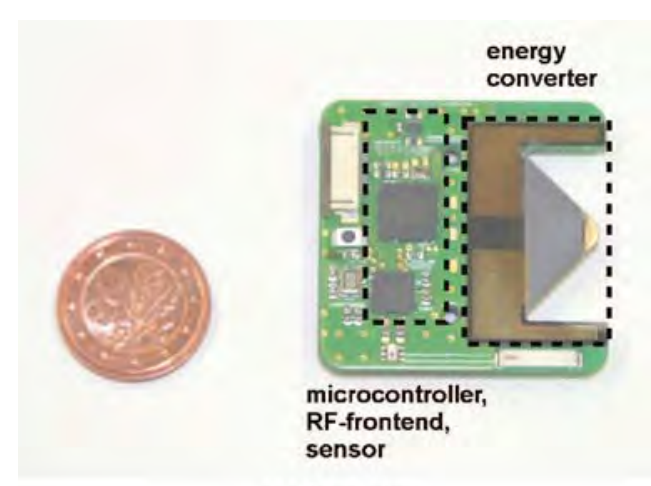

(a) front view

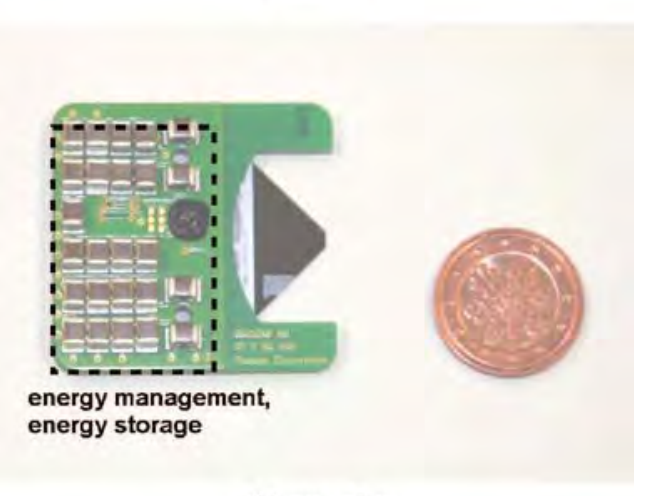

(b) rear view

Fig. 8. Realized energy autonomous sensor node

As stated earlier the MEMS generator until now is not capable of suppling enough voltage. For the evaluation a bulk generator was used.

The RF-front end and the sensor circuitry are connected to the microcontroller. The microcontroller is an 8-bit megaAVR controller from Atmel. It is predestined for use in energy autonomous sensor systems because of its low current consumption in sleep mode. The microcontroller collects the sensor data and forwards it to a base station. This is done wireless via the RF-unit. The sensor node is part of a wireless network therefore the 
communication with the other nodes is defined by interfaces and protocols. The network protocol route under MAC (RUM-protocol) is used.

The storage module of the sensor node has a capacity of $\mathrm{C}_{\mathrm{st}}=2.35 \mathrm{mF}$. Since the bulk generator is used it takes only about $80 \mathrm{~s}$ to load the capacity in the running mode. Meaning to load the difference between the lower voltage of $2.8 \mathrm{~V}$ at which the ASIC switches to load and the higher voltage of $3.5 \mathrm{~V}$ at which it switches to powering the sensor. The initial loading cycle starting with an empty capacity takes considerably longer. The voltage of the sensor transmitting data is shown in Fig. 9. As can be seen the sensor is able to transmit three values with the used capacity size.

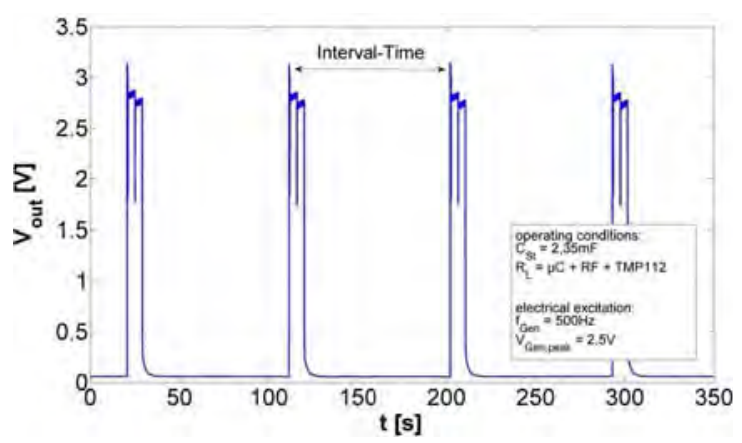

Fig. 9. Voltage across the load

\section{Conclusion}

This paper presented an innovative approach to an autonomous sensor system. It has been shown that the concept of the energy autonomous tire pressure monitoring system powered by a piezoelectric energy harvester itself is feasible. Furthermore the function of its individual components has been shown and evaluated. The next development step will be to construct the whole TPMS in a finished state to be tested under working conditions.

\section{Acknowledgement}

This work is supported by the Federal Ministry of Education and Research (BMBF), Germany and contributes to the project "ASYMOF Autarke Mikrosysteme mit mechanischen Energiewandlern für mobile (Sicherheits-) Funktionen". Reference: 16SV3336 .

\section{References}

[1] A. Frey et al, Autarke Mikrosysteme mit mechanischen Energiewandlern für mobile Sicherheitsfunktionen. 1. Öffentliches Statusmeeting für energieautarke Mikrosysteme, Berlin, 2010.

[2] A. Frey et al., Energy autonomous microsystems for automotive applications, Mikrosystemtechnik Kongress 2011, Darmstadt, Germany, pages 297-300, October 2011.

[3] I. Kuehne et al., Optimum design of a piezoelctrc MEMS generator for fluid actuated energy harvesting, Proceedings MEMS , 2011.

[4] I. Kuehne, Resonate mikromechanische Energiewandler, Shaker Verlag, Aachen, Germany, 2009.

[5] I. Kuehne et al., Fluid-Structure Interaction Modeling for an Optimized Design of a Piezoelectric Energy Harvesting MEMS Generator, Proceedings MEMS, 2011

[6] T. Zimmermann, Entwicklung, Aufbau und Charakterisierung eines kabellosen Sensornetzwerkes basierend auf IEEE 802.15.4-kompatibler Hardware, Hochschule Aalen, Aalen, Germany, July 2010. 